\title{
Long-Term Outcome and Factors Affecting Prognosis of Extracorporeal Shockwave Therapy for Chronic Refractory Achilles Tendinopathy
}

\author{
Joon-Youn Lee, $\mathrm{MD}^{1}$, Kyungjae Yoon, $\mathrm{MD}, \mathrm{PhD}^{1}$, Youbin Yi, $\mathrm{MD}^{1}$, Chul-Hyun Park, MD , \\ Jung-Sang Lee, $\mathrm{MD}^{1}$, Kyoung-Ho Seo, MD, PhD ${ }^{2}$, Young Sook Park, MD, PhD ${ }^{3}$, Yong-Taek Lee, MD, $\mathrm{PhD}^{1}$
}

${ }^{1}$ Department of Physical and Rehabilitation Medicine, Kangbuk Samsung Hospital, Sungkyunkwan University School of Medicine, Seoul; ${ }^{2}$ Department of Physical Medicine and Rehabilitation, Dongtan Sacred Heart Hospital, Hallym University College of Medicine, Hwaseong; ${ }^{3}$ Department of Physical and Rehabilitation Medicine, Samsung Changwon Hospital, Sungkyunkwan University School of Medicine, Changwon, Korea

\begin{abstract}
Objective To investigate the factors affecting prognosis of extracorporeal shockwave therapy (ESWT) for chronic refractory Achilles tendinopathy (AT).

Methods Thirty-six patients (48 consecutive feet) with chronic AT ( $>6$ months) and who underwent ESWT for 'poor' or 'fair' grade in Roles-Maudsley Score (RMS) after unsuccessful conservative treatment were included in the present study. A maximum of 12 sessions of ESWT were conducted until treatment success: RMS reached 'good' or 'excellent'. Termination of ESWT for no response, or 'poor' or 'fair' grade was regarded as treatment failure. Immediate outcome, long-term outcome (telephone interview after mean 26 months), and factors affecting treatment success were analyzed. Results Numeric Rating Scale was significantly decreased at immediate and long-term follow-up. Success rate was $71.1 \%$ and $90.3 \%$, respectively. Univariate logistic regression identified that immediate treatment success was associated with retrocalcaneal enthesophyte on X-ray (odds ratio [OR], 0.06; 95\% confidence interval [CI], 0.01-0.28), pretreatment abnormal ultrasonography echogenicity within Achilles tendon (OR, 18.89; 95\% CI, 2.08-171.96), mean duration of 'post-treatment soreness' (OR, 0.55; 95\% CI, 0.33-0.94), and duration of 'post-treatment soreness after first ESWT' (OR, 0.06; 95\% CI, 0.01-0.34). The duration of 'post-treatment soreness after first ESWT' was found to be the only factor associated with long-term success (OR, 0.32; 95\% CI, 0.10-0.99).

Conclusion ESWT appears to be effective in achieving long-term success in chronic refractory AT. Immediate success was associated with absence of retrocalcaneal enthesophyte on X-ray, presence of pretreatment abnormal ultrasonography echogenicity, shorter mean duration of 'post-treatment soreness', and shorter duration of 'posttreatment soreness after first ESWT. The shorter duration of 'post-treatment soreness after first ESWT' was identified as the only positive prognostic parameter in achieving long-term success.
\end{abstract}

Keywords Tendinopathy, Ultrasonic shockwave, Treatment outcome, Prognosis

Received May 26, 2016; Accepted August 22, 2016

Corresponding author: Yong-Taek Lee

Department of Physical and Rehabilitation Medicine, Kangbuk Samsung Hospital, Sungkyunkwan University School of Medicine, 29 Saemunan-ro, Jongno-gu, Seoul 03181, Korea. Tel: +82-2-2001-2283, Fax: +82-2-2001-1284, E-mail: yongtaek1.lee@samsung.com

ORCID: Joon-Youn Lee (http://orcid.org/0000-0002-2962-527X); Kyung Jae Yoon (http://orcid.org/0000-0002-2765-4309); Youbin Yi (http://orcid. org/0000-0001-5707-3815); Chul-Hyun Park (http://orcid.org/0000-0002-9897-6612); Jung-Sang Lee (http://orcid.org/0000-0001-5423-3772); KyoungHo Seo (http://orcid.org/0000-0002-9139-1638); Young Sook Park (http://orcid.org/0000-0003-2752-7120); Yong-Taek Lee (http://orcid.org/00000001-6611-0999).

(c) This is an open-access article distributed under the terms of the Creative Commons Attribution Non-Commercial License (http://creativecommons.org/ licenses/by-nc/4.0) which permits unrestricted noncommercial use, distribution, and reproduction in any medium, provided the original work is properly cited. Copyright (c) 2017 by Korean Academy of Rehabilitation Medicine 


\section{INTRODUCTION}

Achilles tendinopathy is a clinical condition characterized by pain and tenderness in or around Achilles tendon, occurring frequently in active, as well as, inactive individuals $[1,2]$. Activity modification, stretching, antiinflammatories, physiotherapy, and eccentric loading exercise are known as standard conservative treatment [3-6]. In chronic refractory cases that exhibited no improvement after conservative treatment, surgical intervention has been performed $[4,7,8]$.

Recently, extracorporeal shockwave therapy (ESWT) has emerged as an therapeutic alternative prior to considering surgical option [2,4,7-11]. Despite high evidence of efficacy of ESWT on chronic refractory Achilles tendinopathy [2], some cases still do not respond to ESWT for unknown reasons, causing subjects complain of residual symptoms. Therefore, it becomes important to know which patient is more amenable to ESWT. With regard to standard conservative treatment, Bakkegaard et al. [12] have reported that ultrasonography (US) findings influence the outcome of chronic Achilles tendinopathy. However, no study has investigated the factors associated with the therapeutic outcome of ESWT in patients with chronic refractory Achilles tendinopathy. Therefore, the aim of our study was to investigate the factors influencing outcome of ESWT for chronic refractory Achilles tendinopathy.

\section{MATERIALS AND METHODS}

\section{Subjects}

We evaluated 36 consecutive patients (48 feet) who underwent ESWT for chronic refractory Achilles tendinopathy between 2009 September to 2015 October in Department of Physical and Rehabilitation Medicine at Kangbuk Samsung Hospital. Chronic refractory Achilles tendinopathy was defined as the presence of persistent tenderness on palpation of the tendon, tenosynovium or tendon insertion in patients, which impaired their daily activities for more than 6 months and had not responded to standard conservative treatment. All patients (48 feet) underwent plain radiography. Exclusion criteria consisted of the following: presence of systemic inflammatory disease; previous history of foot surgery; fracture of foot or ankle; and calcification of Achilles tendon. Among 14 patients who had bilateral symptoms, 7 patients showing suspicious features of systemic inflammatory disease, such as morning stiffness and low back pain, underwent further evaluation to rule out systemic inflammatory disease. Three cases were excluded for the following reasons: 1 rheumatoid arthritis; 1 Achilles tendon reconstruction operation; and 1 calcification of Achilles tendon. Finally, 45 feet of 33 patients, who met our eligibility criteria were included in the study. All patients provided informed consent regarding the possibility that their medical records would be used for scientific purposes.

\section{ESWT protocol}

ESWT was indicated if patients had not responded to standard conservative treatment and showed 'poor' or 'fair' grade in the Roles-Maudsley Score (RMS) (Table 1). A maximum of 12 sessions of ESWT $\left(0.10-0.11 \mathrm{~mJ} / \mathrm{mm}^{2}\right.$, 600 shocks, weekly) were conducted until RMS reached 'good' or 'excellent' (treatment success). ESWT was ceased when patients requested for termination of the treatment due to no response to ESWT or increased pain. If 'post-treatment soreness' after ESWT did not subside at subsequent ESWT session, ESWT was postponed until the following week. Patients were educated to perform home-based Achilles tendon stretching exercise, and recommended to reduce activity level as long as possible and avoid impact activities, such as running and jumping. Ten out of 45 feet received additional supervised exercise treatment with conventional physiotherapy. ESWT was performed with EvoTron (SwiTech Medical AG, Kreuzlingen, Switzerland), the electrohydraulic type. Shockwave was applied to the area of maximal tender-

Table 1. Roles and Maudsley score

\begin{tabular}{lcl}
\hline & Point & \multicolumn{1}{c}{ Interpretation } \\
\hline Excellent & 1 & No pain, full movement and activity \\
Good & 2 & Occasional discomfort, full movement and activity \\
Fair & 3 & Some discomfort after prolonged activity \\
Poor & 4 & Pain-limiting activities \\
\hline
\end{tabular}


ness at Achilles tendon with the patient lying in the prone position. The probe with a penetration depth of 5-30 mm was used. The frequency of shock was $1 \mathrm{~Hz}$ and no local anesthesia was applied.

\section{US and MRI evaluation}

Among 45 feet, which were finally included, US was performed in 37 feet and magnetic resonance imaging (MRI) was done in 13 feet for soft tissue evaluation. In addition, both studies were conducted for 5 feet.

Two experienced musculoskeletal radiologists conducted US evaluation of Achilles tendon while patients lying prone with their ankles dorsiflexed $90^{\circ}$. HDI 5000 (Philips Medical Systems, Bothell, WA, USA) and LOGIQ E9 (GE Healthcare, Milwaukee, WI, USA) imaging devices were used for evaluation. The thickness of Achilles tendon was checked at its thickest point, and echogenicity or echotexture was assessed at the entire tendon. The findings were compared with the Achilles tendon of opposite side. US abnormality was defined as thickening of maximum anteroposterior thickness of tendon ( $>6 \mathrm{~mm}$ ) $[13,14]$, or abnormal echogenicity of Achilles tendon (hypoechoic area evident in both the longitudinal and transverse scans and heterogeneous appearance of fibrillar pattern), or hypervascularity within the Achilles tendon or peritendon [12,15-18] (Fig. 1).

MRI examination was performed with a 1.5-T Signa imager (GE Healthcare) using a dedicated surface coil (Medical Advances, Milwaukee, WI, USA). MRI protocols for all subjects were standard. MR images were evaluated on high-resolution monitors of a PACS by above-mentioned two experienced musculoskeletal radiologists. The thickness of Achilles tendon was measured at its thickest point on T1-weighted sagittal view $[14,19]$ for 8 feet which did not undergo US.

\section{Follow-up and clinical assessment}

Pain intensity was measured by Numerical Rating Scale (NRS) before each ESWT session, and at immediate and long-term follow-up. The NRS is an 11-point pain intensity rating scale, where 10 points indicate worst possible pain and 0 point no pain. Immediate follow-up was conducted 1 week after last ESWT. Long-term follow-up was evaluated at mean 26 months after ESWT by telephone interview. RMS assessment was also conducted before the first treatment and at immediate and long-
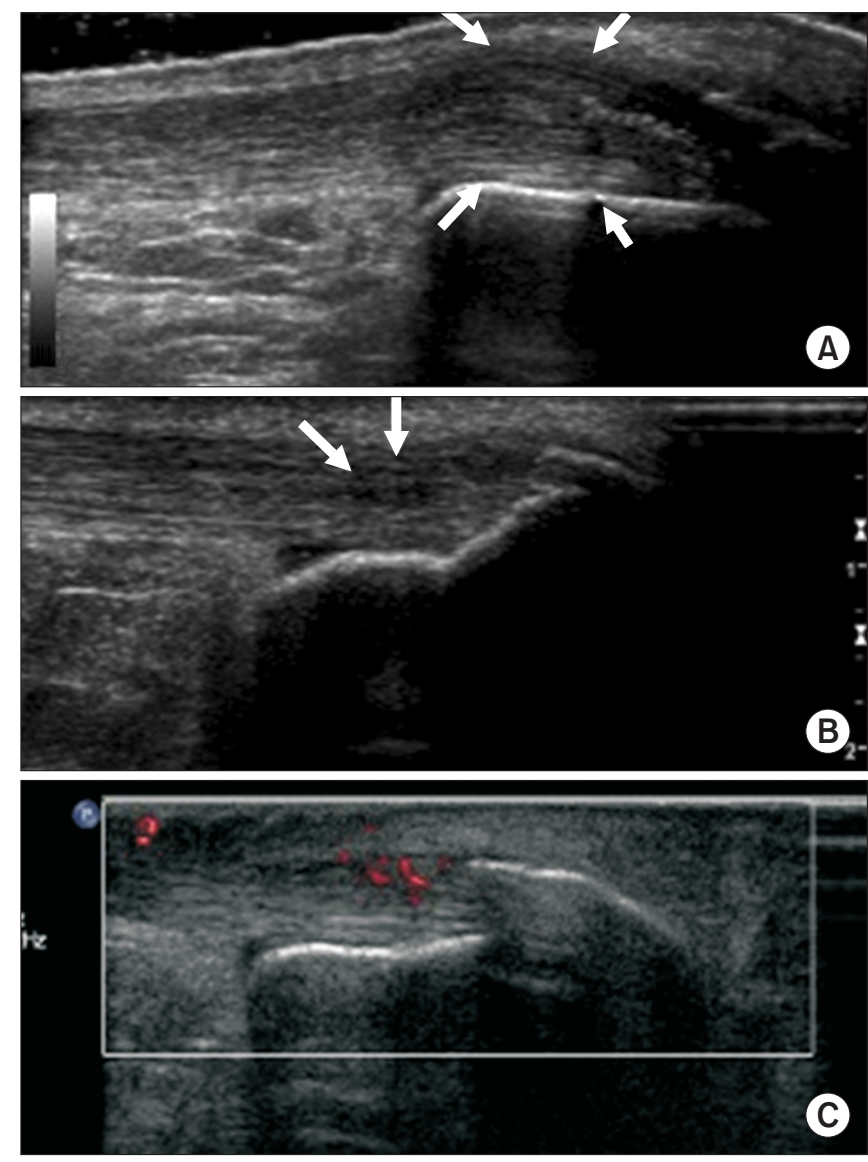

Fig. 1. Ultrasonography abnormality was defined as (A) maximum anteroposterior thickness of tendon $>6 \mathrm{~mm}$, (B) hypoechoic area or heterogeneous appearance of fibrillar pattern, or (C) hypervascularity.

term follow-up, respectively. 'Good' or 'excellent' grade in RMS was regarded as treatment success. Criteria for treatment failure were as follows: cessation of treatment due to no response to ESWT or increased pain; 'poor' or 'fair' grade in RMS at each follow-up. Subjects were divided into two groups: treatment success group and treatment failure group. Factors that can affect outcome of ESWT including age, sex, symptom duration, thickness of Achilles tendon, retrocalcaneal enthesophyte on X-ray, baseline pain intensity (NRS), baseline RMS, total number of ESWT session, mean duration of 'post-treatment soreness', duration of 'post-treatment soreness after first ESWT', additional supervised exercise, previous steroid injection history, and diabetes mellitus (DM) were compared between the groups. US parameters (presence of pretreatment Achilles thickening [ $>6 \mathrm{~mm}$ ], abnormal echogenicity of Achilles tendon, hypervascularity within 
the Achilles tendon or peritendon) were also compared.

\section{Statistical analysis}

Paired t-test was used to analyze the effect of ESWT on pain score (NRS) at immediate and long-term follow-up. Association between 'treatment success' at immediate or long-term follow-up and factors that can affect outcome of ESWT were assessed by univariate logistic regression. Association between immediate treatment success and long-term outcome was analyzed by Fisher exact test. All analyses were performed with the Statistical Package for the Social Sciences (SPSS) software ver. 18.0 (SPSS, Chicago, IL, USA). The $\mathrm{p}$-values $<0.05$ were considered statistically significant.

\section{RESULTS}

A total of 45 feet (33 patients) who met our eligibility criteria were included. Ninety-six percent of them (43 feet, 31 patients) were available for long-term follow-up telephone interview (mean 26 weeks after ESWT). Range of long-term follow-up duration was as follows: 6-12 months in 15 feet; $12-24$ months in 6 feet; 24-36 months in 5 feet; and 36-62 months in 17 feet. A significant decrease in subjective pain was noted in 43 feet at immediate and long-term follow-up, respectively $(\mathrm{p}<0.05)$ (Fig.

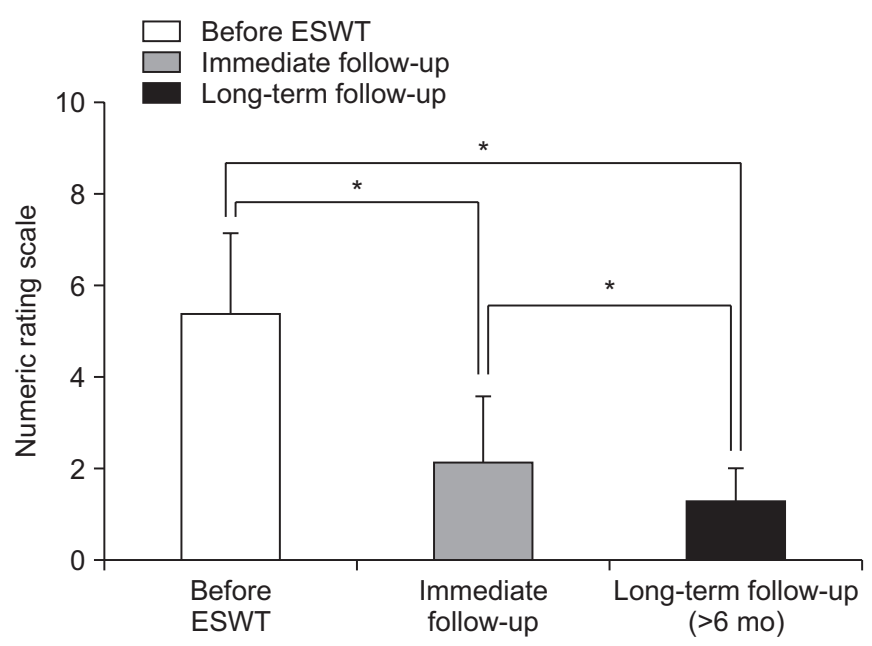

Fig. 2. In 31 patients (43 feet) who were available for longterm follow-up telephone interview, subjective pain was significantly decreased at immediate and long-term followup, respectively $\left({ }^{*} \mathrm{p}<0.05\right)$. ESWT, extracorporeal shockwave therapy.
2). The success rate of ESWT was $71.1 \%$ at immediate and $90.3 \%$ at long-term follow-up. Adverse reactions of the treatment such as bruise or swelling were not found in the present study. Table 2 shows basic characteristics of subjects according to immediate treatment outcome of ESWT. The univariate logistic regression analysis identified that the following factors were associated with immediate treatment success: absence of retrocalcaneal enthesophyte on X-ray; shorter mean duration of 'post-treatment soreness', and shorter duration of 'posttreatment soreness after first ESWT' (Table 3). However, other factors (age, sex, symptom duration, thickness of Achilles tendon, baseline NRS, baseline RMS, additional supervised exercise, previous steroid injection history, and DM) were not significantly associated with immediate treatment outcome (Table 3). Among US parameters, presence of pretreatment abnormal echogenicity of Achilles tendon was significantly associated with immediate treatment success (Table 3 ).

In terms of long-term treatment outcome, duration of 'post-treatment soreness after first ESWT' was found to be the only prognostic factor (odds ratio, 0.32 ; $95 \%$ confidence interval, 0.10-0.99). In other words, shorter duration of 'post-treatment soreness after first ESWT' turned out to be associated with long-term treatment success. In addition, immediate treatment success was significantly associated with long-term treatment success as revealed by Fisher exact test $(\mathrm{p}<0.05)$.

\section{DISCUSSION}

In our study, absence of retrocalcaneal enthesophyte on X-ray was regarded as a positive factor associated with immediate treatment success of ESWT for chronic refractory Achilles tendinopathy. This finding can be explained based on the results of previous studies. Retrocalcaneal enthesophytes may be an adaptive response to increased traction in the Achilles tendon [20,21]. Increased mechanical loading triggers osteoblastic activity at the tendon attachment site, and physically active people are more likely to exhibit presence of enthesophytes [22,23]. Thus, presence of retrocalcaneal enthesophyte may imply that the patient has high activity level and is more liable to overuse injuries. In addition, retrocalcaneal enthesophytes are more common in patients with inflammatory diseases such as spondyloarthropathy as compared to 
Table 2. Basic characteristics of subjects according to immediate treatment outcome of ESWT

\begin{tabular}{|c|c|c|c|c|}
\hline Variable & $\begin{array}{l}\text { Treatment success } \\
(\mathbf{n}=32)\end{array}$ & $\begin{array}{l}\text { Treatment failure } \\
\qquad(n=13)\end{array}$ & $\begin{array}{c}\text { Total } \\
(n=45)\end{array}$ & p-value \\
\hline Age (yr) & $49.4 \pm 9.0$ & $52.2 \pm 9.3$ & $50.2 \pm 9.1$ & $0.356^{\mathrm{a})}$ \\
\hline Sex & & & & $0.538^{b)}$ \\
\hline Male & 13 & 4 & 17 & \\
\hline Female & 19 & 9 & 28 & \\
\hline Symptom duration (day) & $419.0(236.0-1,044.5)$ & $1011.0(387.5-1,517.0)$ & $419.0(328.0-1,141.0)$ & $0.129^{\mathrm{c})}$ \\
\hline Thickness of Achilles tendon (mm) & $5.4(5.0-6.2)$ & $5.1(4.6-5.5)$ & $5.3(4.8-5.8)$ & $0.044^{\mathrm{c})}$ \\
\hline Retrocalcaneal enthesophyte on X-ray & & & & $<0.001^{\text {b) }}$ \\
\hline Presence & 5 & 10 & 15 & \\
\hline Absence & 27 & 3 & 30 & \\
\hline Baseline NRS & $6.0(4.0-6.4)$ & $5.0(3.5-6.5)$ & $5.5(4.0-6.3)$ & $0.294^{\text {b) }}$ \\
\hline Baseline RMS & & & & $0.737^{\mathrm{b})}$ \\
\hline Poor & 19 & 9 & 28 & \\
\hline Fair & 13 & 4 & 17 & \\
\hline Total number of ESWT sessions & $5.5(3.0-6.0)$ & $4.0(2.0-6.0)$ & $5.0(3.0-6.0)$ & $0.307^{\mathrm{c})}$ \\
\hline $\begin{array}{l}\text { Duration of post-treatment soreness } \\
\text { after first ESWT (day) }\end{array}$ & $1.0(0.5-2.0)$ & $3.5(3.0-4.0)$ & $2.0(0.5-3.0)$ & $<0.001^{\mathrm{c})}$ \\
\hline $\begin{array}{l}\text { Mean duration of post-treatment } \\
\text { soreness (day) }\end{array}$ & $1.5(0.5-2.6)$ & $3.0(2.3-3.4)$ & $2.0(0.5-3.1)$ & $0.004^{\mathrm{c})}$ \\
\hline Additional supervised exercise & & & & $0.238^{\mathrm{b})}$ \\
\hline Performed & 9 & 1 & 10 & \\
\hline Not performed & 23 & 12 & 35 & \\
\hline Previous steroid injection history & & & & $0.567^{\mathrm{b})}$ \\
\hline Presence & 2 & 2 & 4 & \\
\hline Absence & 30 & 11 & 41 & \\
\hline Diabetes mellitus & & & & $0.136^{\mathrm{b})}$ \\
\hline Presence & 2 & 3 & 5 & \\
\hline Absence & 30 & 10 & 40 & \\
\hline \multicolumn{5}{|l|}{ Ultrasonography parameter $(\mathrm{n}=37)$} \\
\hline Achilles thickening (>6 mm) & & & & $0.391^{b)}$ \\
\hline Presence & 7 & 1 & 8 & \\
\hline Absence & 19 & 10 & 29 & \\
\hline \multicolumn{2}{|c|}{ Abnormal echogenicity of Achilles tendon } & & & $0.002^{\mathrm{d})}$ \\
\hline Presence & 17 & 1 & 18 & \\
\hline Absence & 9 & 10 & 19 & \\
\hline \multicolumn{3}{|c|}{ Hypervascularity within the Achilles tendon or peritendon } & & $0.540^{\mathrm{b})}$ \\
\hline Presence & 3 & 0 & 3 & \\
\hline Absence & 23 & 11 & 34 & \\
\hline
\end{tabular}

Values are presented as mean \pm standard deviation or median (interquartile range).

NRS, Numeric Rating Scale; RMS, Roles-Maudsley Score; ESWT, extracorporeal shockwave therapy.

${ }^{\text {a) }}$ Student t-test, ${ }^{\text {b) }}$ Fisher exact test, ${ }^{\text {c) }}$ Mann-Whitney U-test, ${ }^{\text {d) }}$ chi-square test. 
Table 3. Association between factors that can affect outcome of ESWT and immediate treatment success

\begin{tabular}{lcc}
\hline \multicolumn{1}{c}{ Variable } & OR (95\% CI) & p-value $^{\text {a) }}$ \\
\hline Age (yr) & $0.97(0.90-1.04)$ & 0.349 \\
Sex (ref. female) & $0.65(0.17-2.56)$ & 0.538 \\
Symptom duration & $1.00(1.00-1.00)$ & 0.098 \\
Thickness of Achilles tendon & $2.64(0.87-8.04)$ & 0.087 \\
Retrocalcaneal enthesophyte on X-ray (ref. absence) & $0.06(0.01-0.28)$ & $<0.001$ \\
Baseline NRS & $1.24(0.83-1.83)$ & 0.294 \\
Baseline RMS-poor (ref. fair) & $1.54(0.39-6.08)$ & 0.538 \\
Total number of ESWT sessions & $1.13(0.88-1.45)$ & 0.341 \\
Duration of post-treatment soreness after first ESWT & $0.06(0.01-0.34)$ & 0.001 \\
Mean duration of post-treatment soreness & $0.55(0.33-0.94)$ & 0.028 \\
Additional supervised exercise (ref. not performed) & $4.70(0.53-41.57)$ & 0.165 \\
Previous steroid injection (ref. absence) & $0.37(0.05-2.93)$ & 0.344 \\
Diabetes mellitus (ref. absence) & $0.22(0.03-1.53)$ & 0.126 \\
Ultrasonography parameter & & \\
Achilles thickening (>6 mm) (ref. absence) & $3.68(0.40-34.29)$ & 0.252 \\
\hline Abnormal echogenicity of Achilles tendon (ref. absence) & $18.89(2.08-171.96)$ & 0.009 \\
\hline Hypervascularity within the Achilles tendon or peritendon (ref. absence) & $1.30(0.12-14.12)$ & 0.827 \\
\hline
\end{tabular}

ESWT, extracorporeal shockwave therapy; OR, odds ratio; CI, confidence interval; NRS, Numeric Rating Scale; RMS, Roles-Maudsley Score.

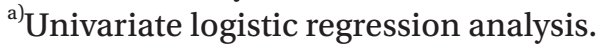

healthy subjects [24-27]. Therefore, it could be possible that subjects with retrocalcaneal enthesophytes have systemic inflammatory diseases such as spondyloarthropathy despite our effort to exclude systemic inflammatory disease.

Presence of 'pretreatment abnormal US echogenicity of Achilles tendon' was found to be another positive parameter associated with immediate treatment success. In terms of standard conservative treatment, there are controversies regarding imaging studies (US or MRI) as a prognostic parameter. Bakkegaard et al. [12] have reported that US findings influence outcome of conservative treatment of chronic Achilles tendinopathy, in which pretreatment abnormal US findings (hyper- or hypoechogenicity) were correlated with poor outcome after 6 months. In contrast, Khan et al. [17] revealed that US changes could not predict 12 months outcome of Achilles tendinopathy. To the best of our knowledge, present study is the first investigation on the association between the outcome of ESWT and US findings in Achilles tendinopathy. Further study on this issue involving large population and prospective study protocol are required.

In our study, the thickness of Achilles tendon was mea- sured at its thickest point (37 feet on US and 8 on MRI). Contrary to our expectation that thickness of Achilles tendon would influence outcome of ESWT, it was intriguing to note that Achilles tendon thickness was not associated with immediate treatment success. This finding is similar to the results of former studies on plantar fasciitis, which stated that thickness of plantar fascia does not influence the outcome of ESWT [28,29]. There have been conflicting results about association of Achilles tendon thickness with symptoms of patient. Some studies have suggested that Achilles tendon thickness is related to the level of pain and dysfunction, and improvement of discomfort is accompanied with reduction in tendon thickness [12,30,31]. On the contrary, Kulig et al. [32] reported that there was no significant difference in thickness of Achilles tendon between asymptomatic and symptomatic dancers.

One major finding of our study, which attracted particular interest, was that, duration of 'post-treatment soreness after first ESWT' was the only one common factor influencing both immediate and long-term treatment success. In our study, all patients had at least half a day of soreness after receiving ESWT, which we defined 
as 'post-treatment soreness'. Pain or tissue damage such as hematoma is a well-known side effect when using ESWT $[33,34]$. Larger energy level seems more likely to be linked with greater pain and tissue damage. For example, capillary disruption and consecutive extravasation of erythrocytes after ESWT may introduce inflammatory reaction and 'post-treatment soreness' [34,35]. In addition, sonographic and histological evaluation showed dosedependent changes in the tendon and paratenon after ESWT ranging from transient swelling of the tendon with a minor inflammatory reaction to paratendinous fluid, marked histological changes with increased eosin staining, fibrinoid necrosis, fibrosis in the paratenon, and infiltration of inflammatory cells. [33-35]. Thus, to some degree, 'post-treatment soreness' could be referred to as a result of appropriate biologic effect caused by appropriate delivery of therapeutic shockwave energy to the targeted area. On the other hand, excessive 'post-treatment soreness' could be a sign of tissue damage caused by over-delivered shockwave energy. Consequently, we authors suggest that duration of 'post-treatment soreness' have a feasibility of use as a parameter to determine appropriate delivery of shock energy.

In our study, a maximum of 12 sessions of ESWT (0.10$0.11 \mathrm{~mJ} / \mathrm{mm}^{2}, 600$ shocks, weekly) were conducted until RMS reached 'good' or 'excellent' (treatment success). Our ESWT protocol is the modified version of the protocol used in previous well-designed studies $[4,9,36,37]$. In the reported studies, Furia et al. [4,37] performed just a single session of ESWT using high-energy $\left(0.21 \mathrm{~mJ} / \mathrm{mm}^{2}\right)$ with 3,000 shocks; while Rompe et al. [36] executed 3 sessions of ESWT using low-energy $\left(0.12 \mathrm{~mJ} / \mathrm{mm}^{2}\right)$ with 2,000 shocks per session. Total effective treatment energy is usually defined as energy flux density multiplied by number of shocks. In our protocol, although total effective treatment energy (maximum $720-792 \mathrm{~mJ} / \mathrm{mm}^{2}$ ) was similar to those of previous studies $\left(630 \mathrm{~mJ} / \mathrm{mm}^{2}\right.$ in Furia et al. [4,37]; $720 \mathrm{~mJ} / \mathrm{mm}^{2}$ in Rompe et al. [36]), total effective treatment energy per each treatment session was much less $\left(60-66 \mathrm{~mJ} / \mathrm{mm}^{2}\right)$ than the values reported by Furia et al. $\left(630 \mathrm{~mJ} / \mathrm{mJ} / \mathrm{mm}^{2}\right)$ and Rompe et al. $\left(240 \mathrm{~mJ} / \mathrm{mm}^{2}\right)$.

We tried to avoid excessive delivery of energy to a targeted area per each treatment session. Therefore, to reach total effective treatment energy comparable to those of former studies, more treatment sessions (up to a maximum of 12 sessions) than former studies (one ses- sion in Furia et al.; three in Rompe et al.) were inevitably required.

Among 33 patients ( 45 feet) who met our eligibility criteria, 43 feet were available for long-term follow-up telephone interview and long-term follow-up rate was $96 \%$. At both immediate follow-up (1 week after last ESWT) and long-term follow-up (mean 26 weeks after ESWT), significant decrease in subjective pain was noted, respectively ( $\mathrm{p}<0.05)$. The immediate success rate of ESWT was $71.1 \%$ and long-term success rate was $90.3 \%$. Although the outcome measures for clinical assessment were different, these results are similar to those published in previous randomized controlled trials, which reported ESWT as an effective method of treatment in chronic recalcitrant tendinopathy $[9,36,38]$. Meanwhile, success rates in our study were found to be generally higher than previously reported studies $[4,9,36,37]$. We believe that such results could be attributed to our efforts to reduce excessive 'post-treatment soreness' as follows: taking anti-inflammatory medication and applying ice massage before beginning of ESWT to reduce possible concomitant acute inflammatory pain; use of low-energy flux density $\left(0.10-0.11 \mathrm{~mJ} / \mathrm{mm}^{2}\right)$ and less total effective treatment energy per each treatment session $\left(60-66 \mathrm{~mJ} / \mathrm{mm}^{2}\right)$ to avoid excessive delivery of energy to a targeted area per each treatment session; and postponement of ESWT until the following week if 'post-treatment soreness' did not subside. Actually, the notion whether ESWT was successful or not at immediate follow-up was significantly associated with long-term success of ESWT in the present study.

There were some limitations in our study that should be discussed. Firstly, small number of subjects may have reduced statistical power of the results. Especially, at long-term follow-up, only four cases were included in the treatment failure group because most of the cases (39 feet of 43) turned out to be 'treatment success', which made investigation of the true factors that can affect long-term outcome of ESWT a difficult task. Secondly, retrospective data collection via chart review may have resulted in generation of incomplete information. Past medical history other than DM and anthropometric data, such as body mass index, could not be evaluated due to lack of medical records. Lastly, we could not consider activity level which is regarded as a contributor to Achilles tendinopathy $[39,40]$, since the afore-mentioned information were 
not available. Nevertheless, we believe that activity level had little impact on our results because all subjects were recommended to reduce their activity level for as long as possible and avoid impact activities. Further studies are needed to supplement these limitations.

In conclusion, low-energy ESWT appears to be an effective treatment option for chronic refractory Achilles tendinopathy while considering long-term follow-up. Immediate treatment success was associated with shorter mean duration of 'post-treatment soreness', shorter duration of 'post-treatment soreness after first ESWT', absence of retrocalcaneal enthesophyte on X-ray, and presence of pretreatment abnormal US echogenicity of AT. In case of long-term success, 'post-treatment soreness after first ESWT' was found to be the only positive prognostic parameter.

\section{CONFLICT OF INTEREST}

No potential conflict of interest relevant to this article was reported.

\section{ACKNOWLEDGMENTS}

The authors would like to take this opportunity to express their special thanks to Ms. Miyeon Lee at the medical information library in Kangbuk Samsung Hospital for her advice about statistical analysis of the current study.

\section{REFERENCES}

1. Lake JE, Ishikawa SN. Conservative treatment of Achilles tendinopathy: emerging techniques. Foot Ankle Clin 2009;14:663-74.

2. Gerdesmeyer L, Mittermayr R, Fuerst M, Al Muderis M, Thiele R, Saxena A, et al. Current evidence of extracorporeal shock wave therapy in chronic Achilles tendinopathy. Int J Surg 2015;24(Pt B):154-9.

3. Alfredson H, Lorentzon R. Chronic Achilles tendinosis: recommendations for treatment and prevention. Sports Med 2000;29:135-46.

4. Furia JP. High-energy extracorporeal shock wave therapy as a treatment for insertional Achilles tendinopathy. Am J Sports Med 2006;34:733-40.

5. Den Hartog BD. Insertional Achilles tendinosis: pathogenesis and treatment. Foot Ankle Clin
2009;14:639-50.

6. Almekinders LC. Tendinitis and other chronic tendinopathies. J Am Acad Orthop Surg 1998;6:157-64.

7. Lakshmanan P, O'Doherty DP. Chronic Achilles tendinopathy: treatment with extracorporeal shock waves. Foot Ankle Surg 2004;10:125-30.

8. Taylor J, Dunkerley S, Silver D, Redfern A, Talbot N, Sharpe I, et al. Extracorporeal shockwave therapy (ESWT) for refractory Achilles tendinopathy: a prospective audit with 2-year follow up. Foot (Edinb) 2016;26:23-9.

9. Rompe JD, Nafe B, Furia JP, Maffulli N. Eccentric loading, shock-wave treatment, or a wait-and-see policy for tendinopathy of the main body of tendo Achillis: a randomized controlled trial. Am J Sports Med 2007;35:374-83.

10. Mani-Babu S, Morrissey D, Waugh C, Screen H, Barton $\mathrm{C}$. The effectiveness of extracorporeal shock wave therapy in lower limb tendinopathy: a systematic review. Am J Sports Med 2015;43:752-61.

11. Al-Abbad H, Simon JV. The effectiveness of extracorporeal shock wave therapy on chronic achilles tendinopathy: a systematic review. Foot Ankle Int 2013;34:33-41.

12. Bakkegaard M, Johannsen FE, Hojgaard B, Langberg $\mathrm{H}$. Ultrasonography as a prognostic and objective parameter in Achilles tendinopathy: a prospective observational study. Eur J Radiol 2015;84:458-62.

13. Van Holsbeeck M, Introcaso JH. Musculoskeletal ultrasound. St. Louis: Mosby; 1991.

14. Richards PJ, Dheer AK, McCall IM. Achilles tendon (TA) size and power Doppler ultrasound (PD) changes compared to MRI: a preliminary observational study. Clin Radiol 2001;56:843-50.

15. Kainberger FM, Engel A, Barton P, Huebsch P, Neuhold A, Salomonowitz E. Injury of the Achilles tendon: diagnosis with sonography. AJR Am J Roentgenol 1990;155:1031-6.

16. Kallinen M, Suominen H. Ultrasonographic measurements of the Achilles tendon in elderly athletes and sedentary men. Acta Radiol 1994;35:560-3.

17. Khan KM, Forster BB, Robinson J, Cheong Y, Louis L, Maclean L, et al. Are ultrasound and magnetic resonance imaging of value in assessment of Achilles tendon disorders? A two year prospective study. Br J Sports Med 2003;37:149-53. 
18. Koivunen-Niemela T, Parkkola K. Anatomy of the Achilles tendon (tendo calcaneus) with respect to tendon thickness measurements. Surg Radiol Anat 1995;17:263-8.

19. Soila K, Karjalainen PT, Aronen HJ, Pihlajamaki HK, Tirman PJ. High-resolution MR imaging of the asymptomatic Achilles tendon: new observations. AJR Am J Roentgenol 1999;173:323-8.

20. Benjamin M, Ralphs JR. The cell and developmental biology of tendons and ligaments. Int Rev Cytol 2000; 196:85-130.

21. Benjamin M, Rufai A, Ralphs JR. The mechanism of formation of bony spurs (enthesophytes) in the achilles tendon. Arthritis Rheum 2000;43:576-83.

22. Kannus P, Jozsa L, Kvist M, Jarvinen TL, Maunu VM, Hurme T, et al. Expression of osteocalcin in the patella of experimentally immobilized and remobilized rats. J Bone Miner Res 1996;11:79-87.

23. Tyrdal S, Finnanger AM. Osseous manifestations of 'handball goalie's elbow'. Scand J Med Sci Sports 1999; 9:92-7.

24. Girolomoni G, Gisondi P. Psoriasis and systemic inflammation: underdiagnosed enthesopathy. J Eur Acad Dermatol Venereol 2009;23 Suppl 1:3-8.

25. Feydy A, Lavie-Brion MC, Gossec L, Lavie F, Guerini H, Nguyen C, et al. Comparative study of MRI and power Doppler ultrasonography of the heel in patients with spondyloarthritis with and without heel pain and in controls. Ann Rheum Dis 2012;71:498-503.

26. Aydin SZ, Can M, Alibaz-Oner F, Keser G, Kurum E, Inal V, et al. A relationship between spinal new bone formation in ankylosing spondylitis and the sonographically determined Achilles tendon enthesophytes. Rheumatol Int 2016;36:397-404.

27. Genc H, Cakit BD, Tuncbilek I, Erdem HR. Ultrasonographic evaluation of tendons and enthesal sites in rheumatoid arthritis: comparison with ankylosing spondylitis and healthy subjects. Clin Rheumatol 2005;24:272-7.

28. Chuckpaiwong B, Berkson EM, Theodore GH. Extracorporeal shock wave for chronic proximal plantar fasciitis: 225 patients with results and outcome predictors. J Foot Ankle Surg 2009;48:148-55.

29. Maki M, Ikoma K, Imai K, Kido M, Hara Y, Arai Y, et al. Correlation between the outcome of extracorporeal shockwave therapy and pretreatment MRI findings for chronic plantar fasciitis. Mod Rheumatol 2015;25:42730.

30. Ohberg L, Alfredson H. Effects on neovascularisation behind the good results with eccentric training in chronic mid-portion Achilles tendinosis? Knee Surg Sports Traumatol Arthrosc 2004;12:465-70.

31. Ohberg L, Lorentzon R, Alfredson H. Eccentric training in patients with chronic Achilles tendinosis: normalised tendon structure and decreased thickness at follow up. Br J Sports Med 2004;38:8-11; discussion 11.

32. Kulig K, Oki KC, Chang YJ, Bashford GR. Achilles and patellar tendon morphology in dancers with and without tendon pain. Med Probl Perform Art 2014;29:2218.

33. Haake M, Boddeker IR, Decker T, Buch M, Vogel M, Labek G, et al. Side-effects of extracorporeal shock wave therapy (ESWT) in the treatment of tennis elbow. Arch Orthop Trauma Surg 2002;122:222-8.

34. Peters J, Luboldt W, Schwarz W, Jacobi V, Herzog C, Vogl TJ. Extracorporeal shock wave therapy in calcific tendinitis of the shoulder. Skeletal Radiol 2004;33:7128.

35. Rompe JD, Kirkpatrick CJ, Kullmer K, Schwitalle M, Krischek O. Dose-related effects of shock waves on rabbit tendo Achillis: a sonographic and histological study. J Bone Joint Surg Br 1998;80:546-52.

36. Rompe JD, Furia J, Maffulli N. Eccentric loading compared with shock wave treatment for chronic insertional Achilles tendinopathy: a randomized, controlled trial. J Bone Joint Surg Am 2008;90:52-61.

37. Furia JP. High-energy extracorporeal shock wave therapy as a treatment for chronic noninsertional Achilles tendinopathy. Am J Sports Med 2008;36:502-8.

38. Rasmussen S, Christensen M, Mathiesen I, Simonson O. Shockwave therapy for chronic Achilles tendinopathy: a double-blind, randomized clinical trial of efficacy. Acta Orthop 2008;79:249-56.

39. Sobhani S, Dekker R, Postema K, Dijkstra PU. Epidemiology of ankle and foot overuse injuries in sports: a systematic review. Scand J Med Sci Sports 2013;23:669-86.

40. Filardo G, Kon E, Di Matteo B, Di Martino A, Tesei G, Pelotti $P$, et al. Platelet-rich plasma injections for the treatment of refractory Achilles tendinopathy: results at 4 years. Blood Transfus 2014;12:533-40. 